\title{
Misplaced Ventricular Lead via an Atrial Septal Defect-Percutaneous Extraction
}

\author{
${ }^{1}$ Department of Cardiac Surgery, University Clinic Oldenburg, \\ European Medical School Oldenburg-Groningen, Oldenburg, \\ Germany \\ 2 Department of Anaesthesiology, Critical Care, Emergency Medicine \\ and Pain Therapy, University Clinic Oldenburg, European Medical \\ School Oldenburg-Groningen, Oldenburg, Germany \\ ${ }^{3}$ Department for Cardiovascular Surgery, University Hospital \\ Hamburg, Hamburg, Germany \\ Thorac Cardiovasc Surg Rep 2019;8:e8-e10.
}

Jerry Easo ${ }^{10}$ Malte Book ${ }^{2}$ Samer Hakmi ${ }^{3}$ Alexander Weymann ${ }^{1}$

\author{
Address for correspondence Jerry Easo, MD, Department of Cardiac \\ Surgery, University Clinic Oldenburg, European Medical School \\ Oldenburg-Groningen, Rahel Straus Street 10, Oldenburg 26133, \\ Germany (e-mail: easo.jerry@klinikum-oldenburg.de).
}

\author{
Abstract \\ Keywords \\ - lead extraction \\ - misplaced ventricular \\ lead \\ - mitral valve \\ regurgitation
}

Background Necessity for lead removal in this case was after 12 years due to mitral valve regurgitation caused by retraction of the posterior leaflet by the inadvertently misplaced lead.

Case Description This history describes a 45-year-old woman with history of multiple cardiac operations at young age with an abandoned defibrillator lead via a patent atrial septal defect. Lead extraction was performed with first described use of rotational dilating sheaths to reduce emboli risk, hoping to avoid a fourth surgical procedure with high risk.

Conclusions Percutaneous lead removal using rotational sheaths is possible even for misplaced leads after long-time intervals with acceptable operative risk.

\section{Introduction}

Lead extraction plays an increasingly important role in the era of congestive heart failure with CIED (cardiovascular implantable electronic device) treatment. Misplaced leads are uncommon but have been described in the past, most often via patent atrial septal defects or by iatrogenic perforation. ${ }^{1-4}$ Lead extraction is described in an early time period, late extraction not recommended due to thromboembolic risk. Laser extraction has been described but should not be the first option to choose, ${ }^{5,6}$ the authors have opted for rotational dilating sheaths for extraction in the attempt to avoid a reoperative surgical procedure for the lead induced mitral valve regurgitation.

(1DJerry Easo's ORCID is https://orcid.org/0000-0001-9445-5716.

\section{Case History}

In this article, the authors present images of a 45-year-old female patient with history of three cardiac surgery procedures, including ventricular septal defect (VSD) closure at the age of 1 year and re-VSD closure at 3 years. Single chamber implantable cardioverter-defibrillator (ICD) implantation was performed due to ventricular tachycardia in 2006. Right branch bundle block and echocardiographic examination ultimately led to diagnosis of an inadvertent placement of the single coil ICD lead over a patent atrial septal defect and passage over the mitral valve, causing regurgitation due to retraction of the posterior leaflet ( $\boldsymbol{- F i g . 1 A - D ;} \boldsymbol{\sim}$ Videos 1 and 2). Abandonment of the left ventricular lead and placement of a right ventricular lead was performed in 2012. Recurrent bouts of dyspnoea and decompensation demonstrated mitral regurgitation to be etiologic of the condition. Due to the highrisk profile of the patient requiring a fourth surgical
License terms

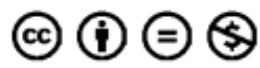

received

December 4, 2018 accepted after revision January 24, 2019
DOI https://doi.org/

10.1055/s-0039-1687821. ISSN 2194-7635. (c) 2019 Georg Thieme Verlag KG Stuttgart · New York 


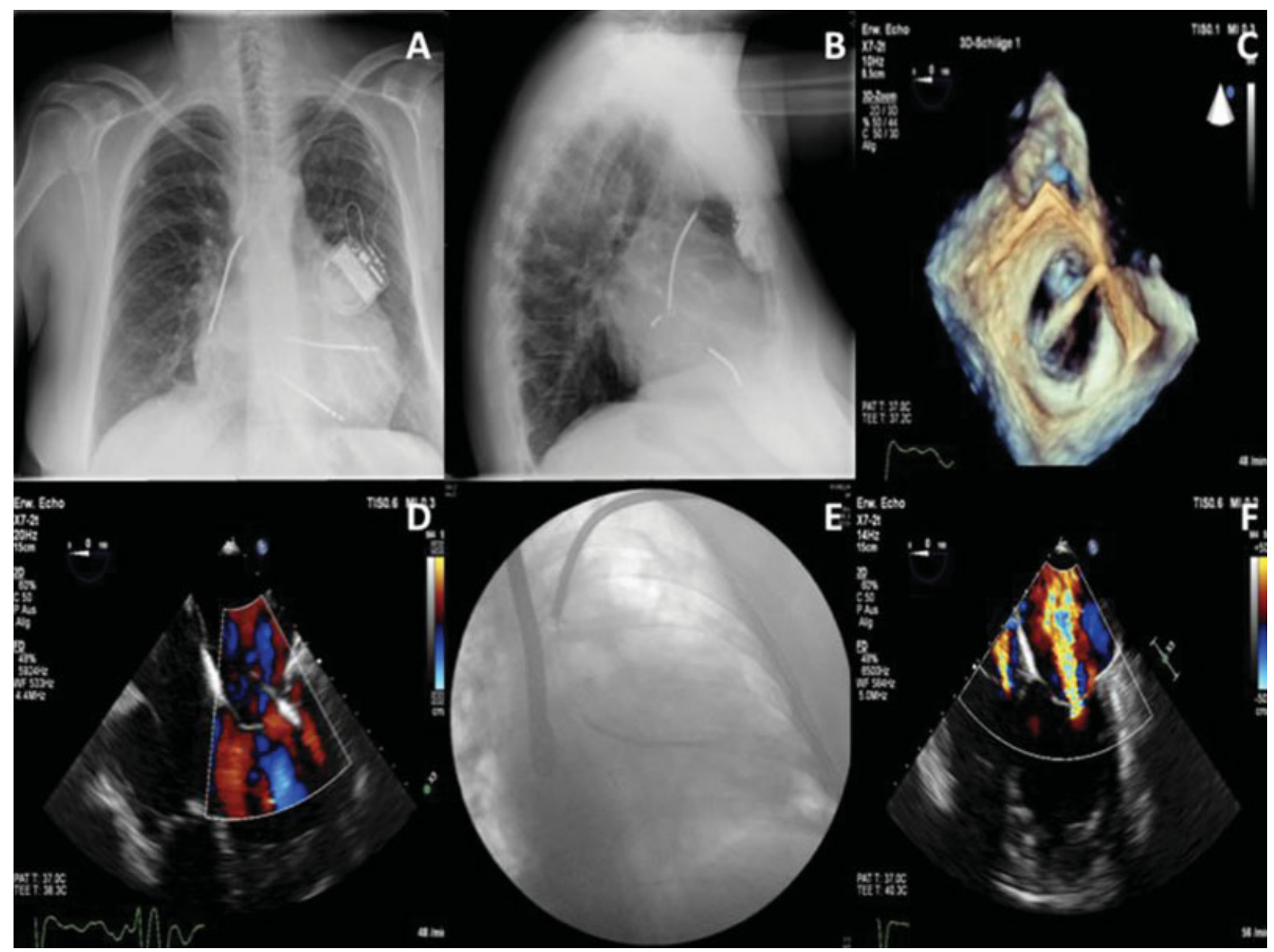

Fig. 1 (A) Posterioranterior view of the single chamber implantable cardioverter-defibrillator with abandoned left ventricular lead and correctly placed right ventricular lead. (B) Lateral chest view of the single chamber ICD. (C) 3D transoesophageal echocardiographic view demonstrating passage of misplaced left ventricular lead over patent atrial septal defect and retraction of the posterior mitral leaflet. (D) 2D transoesophageal echocardiographic view demonstrating mitral regurgitation by retraction of the posterior mitral leaflet with moderate/large jet extending up to the pulmonary veins. (E) Posterior-anterior view of the Spectranetics Tightrail 11 French with adhesiotomy of the left ventricular lead up to the right atrium after successful extraction of the right ventricular lead. (F) 2D transoesophageal echocardiographic view demonstrating similar mitral regurgitation by retraction and fibrosis of the posterior leaflet with moderate/large jet extending up to the pulmonary veins after lead extraction. 2D, two-dimensional; 3D, three-dimensional.

procedure, the authors attempted to improve the mitral pathology by extraction of the retracting ICD lead. Lead removal was performed using percutaneous rotating dilator sheaths (Spectranetic Tightrail 11-F); the option of laser photoablation was abandoned due to laser-energy-induced microbubble formation with increased emboli risk via the patent septal defect. This procedure was performed with cardiopulmonary bypass standby; adhesiotomy up to the cavity of the right atrium could be achieved with echocardiographic support to rule out effusions or pericardial tamponade ( $\mathbf{- F i g}$. 1E). Lead extraction was successful, the retraction of the posterior leaflet after 12 years, however, was caused fibrosis and valve regurgitation remained (-Fig. 1F). Due to lack of immediate hemodynamic compromise surgical mitral valve repair and closure of the atrial septal defect could be planned; percutaneous treatment by Mitraclip was unfortunately not possible due to the morphologic damage to the posterior mitral leaflet and lack of coaptation. Mitral valve replacement was performed 4 weeks later due to worsening hemodynamics, with demise of the patient after a complicated postoperative recovery due to cardiogenic compromise 2 months postsurgery.

\section{Video 1}

2D transoesophageal echocardiography demonstrating mitral regurgitation by retraction of the posterior mitral leaflet by passage of the ICD lead into the left ventricle. 2D, two-dimensional. Online content including video sequences viewable at: https://www.thieme-connect.com/products/ ejournals/html/10.1055/s-0039-1687821. 


\section{Discussion}

Tricuspid valve regurgitation by ICD lead passage is a common phenomenon encountered by experienced CIED surgeons with increasing referral for lead extraction to improve valve competency. ${ }^{7}$ Small case series demonstrate up to $10 \%$ of patients returning to normal tricuspid valve function after removal of leads. ${ }^{8}$ Misplaced leads over septal defects passing to the left heart are uncommon but have been reported in the past, seldom with such compromised mitral valve function as in this case ${ }^{4}$. Anticoagulation with warfarin is recommended for patients not undergoing any other form of simultaneous cardiac surgery ${ }^{2-4}$; lead extraction not recommended due to embolic risk. When extraction is necessary, this often performed in combination with other open heart surgery procedures. Successful lead extraction is described within an early time span when performed; single case histories for longer time spans report on lead extraction using varying tools. ${ }^{5,6}$ The images presented in this case study demonstrate the first described successful use of rotating dilator sheaths for extraction of such a misplaced lead via the septal defect after such a long time interval of 12 years. Three-dimensional (3D) echocardiographic imaging provided valuable information for planning and implementation of the percutaneous procedure (-Video 2).

\section{Video 2}

3D transoesophageal echocardiography demonstrating mitral valve pathology. 3D, threedimensional. Online content including video sequences viewable at: https://www.thieme-connect. com/products/ejournals/html/10.1055/s-00391687821.

Unfortunately, the goal of avoiding open surgery could not be prevented by the extraction; retrospectively one can argue that an open procedure could have been performed as the primary procedure. The operative risk, however, was high as a repeat procedure and was therefore chosen after conferring with the initially hesitant patient. We chose to extract the lead, hoping that the $10 \%$ improvement in valve function reported in other series would apply in our case as well. Unfortunately, this was not the case. However, the initial clinical success of lead extraction underscored our rationale utilizing a low risk procedure first despite greater uncertainty of success. The time interval of 4 weeks after lead extraction was utilized for improving the cardiopulmonary situation of the high-risk patient with recompensation and inotrope treatment. Mitral valve replacement was necessary due to grave morphological alteration of the leaflets with no possibility of reconstruction; unfortunately, the high-risk surgery could not hinder the demise of the patient after an eventful postoperative treatment.

Conflict of Interest

None.

\section{References}

1 Wilkoff BL, Love CJ, Byrd CL, et al; Heart Rhythm Society; American Heart Association. Transvenous lead extraction: Heart Rhythm Society expert consensus on facilities, training, indications, and patient management: this document was endorsed by the American Heart Association (AHA). Heart Rhythm 2009;6 (07):1085-1104

2 Van Gelder BM, Bracke FA, Oto A, et al. Diagnosis and management of inadvertently placed pacing and ICD leads in the left ventricle: a multicenter experience and review of the literature. Pacing Clin Electrophysiol 2000;23(05):877-883

3 Rodriguez Y, Baltodano P, Tower A, Martinez C, Carrillo R. Management of symptomatic inadvertently placed endocardial leads in the left ventricle. Pacing Clin Electrophysiol 2011;34(10):1192-1200

4 Aquila I, García A, Fernández-Golfín C, Zamorano JL. It paces right but not in the right space!. Eur Heart J 2016;37(05):496

5 Anastacio MM, Castillo-Sang M, Lawton JS. Laser extraction of pacemaker lead traversing a patent foramen ovale and the mitral valve. Ann Thorac Surg 2012;94(06):2125-2127

6 Nägele H, Schneider JC, Knobelsdorff GV, Petersen B, Rödiger W. Excimer laser-assisted extraction of an infected bipolar left ventricular pacing lead implanted 10 years ago. Pacing Clin Electrophysiol 2001;24(03):388-390

7 Al-Mohaissen MA, Chan KL. Prevalence and mechanism of tricuspid regurgitation following implantation of endocardial leads for pacemaker or cardioverter-defibrillator. J Am Soc Echocardiogr 2012;25 (03):245-252

8 Pecha S, Castro L, Gosau N, et al. Evaluation of tricuspid valve regurgitation following laser lead extraction $\dagger$. Eur J Cardiothorac Surg 2017;51(06):1108-1111 